\title{
COUPLED HEURISTIC AND SIMULATION SCHEDULING IN A HIGHLY VARIABLE ENVIRONMENT
}

\author{
Olga Bagatourova \\ Sudhakar K. Mallya \\ Bank of America \\ 1401 Elm Street \\ Dallas, TX 75202, U.S.A.
}

\begin{abstract}
The topic of this paper is workforce scheduling in a highly variable environment - check proofing and encoding in a bank - where uncertainties include both volume and arrival pattern of the work. A shop floor simulation model and a heuristic algorithm for workforce scheduling using simulation to estimate objective function are presented. The proposed approach results in a robust workforce schedule that can be easily adjusted in real time in response to environmental changes, such as volume and throughput fluctuations. A planning and scheduling framework within which the simulation is implemented is briefly discussed.
\end{abstract}

\section{INTRODUCTION}

Check processing represents an essential part of payment services provided by a bank. The process starts with proofing and encoding - replacing hand-written information with MICR (Magnetic Inc Character Recognition) line and correcting errors that could be caused either by customers or tellers. Because checks arriving from other banks are already encoded and big wholesale customers often pre-encode their checks as well, only a small percent of the total checks volume requires this first step. Nevertheless, a proof site processes anywhere from 100,000 to 2,000,000 checks a day and the total number of checks that are proofed and encoded equals several billions items per year.

After the checks are proofed they are imaged, sorted and those that are drawn on other banks are bundled and dispatched to Federal Reserve or directly to the correspondent bank for collection. Missed dispatch times and deadlines turn into lost funds availability and higher Federal Reserve clearance fees for the bank. Since proof is the first step in the process it is crucial to meet the most favorable deadlines.
Proof operation managers are challenged by the following inherent variabilities:

- Volume. The volume of work varies significantly (up to two times) depending on the day of the week, month of the year, holiday on the following or preceding day, consecutive number of the week in the month, pay days in the area and other factors. Forecasting the expected volume is a problem in its own right (see Boyd and Mabert 1977) and is not covered in this paper. Here we will assume that forecasts based on historic data may over- or underestimate the actual volume by as much as $15 \%$.

- Work Arrival Pattern. The work arrival pattern is largely determined by the truck schedules and is affected by traffic, weather conditions, amount of work coming from different sources, etc. Arrival times and weights of incoming work are captured by Receive Sentry ${ }^{\circledR}$ system. Volumes are estimated through the application of a site specific weight to volume ratio. This introduces an additional element of stochasticity in an already variable environment.

- Productivity. Workforce turnover in proof operations is relatively high due to the part-time nature of the work and the fact that it attracts college students or individuals seeking to supplement their incomes. The productivity of individual encoders varies from 700 checks per hour for new hires to 3,500 checks per hour for experienced operators. Consequently, workforce schedules need to take into consideration the variability in productivity and individual preferences among others without compromising safety, service and productivity goals. This proves to be a challenge, especially for large facilities where the operator count may exceed 250 operators.

When scheduling the proof shop workforce the manager has to make decisions on the start and end times for the operators for each day of the week. The roster has to be built in such 
way as to provide sufficient capacity to process the work on time (meet internal deadlines) while minimizing idle time or excess capacity. In addition, a manager can benefit from real time predictive capabilities of the decision support tool e.g. bringing in additional operators if the actual volume is unexpectedly high or letting some of the operators leave earlier if there is not enough work for all the operators on the floor.

Deterministic formulation of the problem is NP hard and does not capture the inherent variability of the underlying process. A corresponding stochastic formulation (Mabert 1979) is as difficult to solve and at the same time does not guarantee a reasonable solution for the worst case. None of the above approaches incorporate individual preferences and constraints of the operators resulting in an unrealistic schedule.

Simulation and heuristics prove to be adequate tools for building a "workable" close to optimal weekly schedule. Two types of simulation models were developed and tested. The first model incorporated all the details of the proof and encoding operations including shop floor layout, transportation of bundles of checks to the work stations, correction of errors, etc. The model was built in Arena (Mallya and Modi 2003). The second simplified model was built in MS-Excel ${ }^{\circledR}$ which assumed that the arriving work was immediately available to the operators. The results of Arena simulation in terms of estimated completion time were very close to the results of Excel ${ }^{\circledR}$ simulation. The simplified Excel model underestimated completion times (time when all work is completed) by 5 to 10 minutes because of the modeling abstractions and assumptions. Specifically, the Excel ${ }^{\circledR}$ based model assumes that there are no delays between receipt of the work and its availability at the proof work station. Additionally, it assumes that check bundles are of the same size and are distributed equally among the associates on the shop floor during that time interval. Notwithstanding these simplifications, the results of both models are within acceptable error levels. Consequently, the Excel ${ }^{\circledR}$ model was selected as the basis for schedule optimization heuristics.

\section{PLANNER AND SCHEDULER FRAMEWORK}

The developed tool includes several features that are designed to support management decision making - forecasting of the volume, resource planning \& scheduling and real time tracking of actual completion. Although this paper deals only with one aspect within the overall framework of the tool, namely, staff scheduling, we will briefly describe the tool architecture in order to place scheduling in the context of proof shop floor management. Figure 1 illustrates the functionality and high-level architecture of the developed framework.

The historic data on planned and actual work volumes, arrival patterns as well as operators' productivities and schedules are stored in the database. The database is configured to interface with other legacy systems for data ex change - the user can import data from reports generated

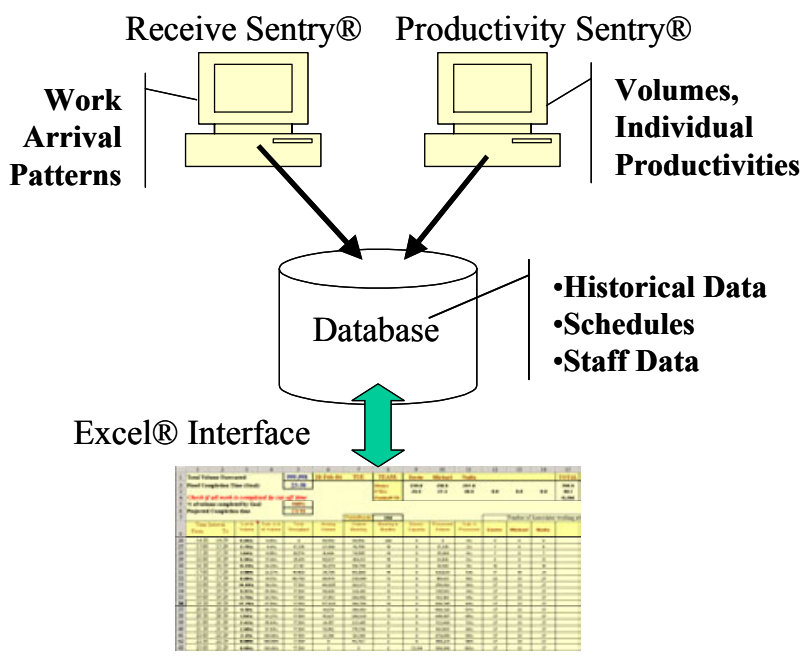

Figure 1: Planning and Scheduling Framework

by stand-alone systems monitoring work arrival (Receive Sentry ${ }^{\circledR}$ ) and productivity (Productivity Sentry ${ }^{\circledR}$ ). Actual volume entered by the manager at the end of the day provides historical data for developing forecasts.

Planning for the week involves forecasting daily volumes, updating operators' schedules with current attendance information and adjusting these schedules to satisfy deadlines and minimize idle or excess capacity. The scheduling of workforce is the focus of this paper and will be described in details in section 3 .

The simulation model is built in Excel ${ }^{\circledR}$ and is invoked every time the user wishes to assess the results of environment changes. The environmental components are:

- Total volume

- Work arrival pattern

- Actual attendance of the operators

The simulation results are presented to the user as a table organized by half hour intervals (rows) and display information on the available throughput, amount of arriving work, amount of WIP on the floor, excess capacity, headcount, etc. (columns). A snapshot of the table can be seen at the bottom of Figure 1 as an example of Excel user interface. The tool also provides the user with several graphs depicting simulation results. In Figure 2 the $\mathrm{x}$ axis is labeled with times of the day and the y axis is labeled with the number of fields. The red bars are the WIP on the floor, the blue bars are total available throughput and the lilac bars represent the excess capacity.

The workbook is configured to represent a whole week with a separate spreadsheet for each day of the week. It also contains a separate spreadsheet with a summary of the weekly schedule. This is used to communicate schedule to the operators. 


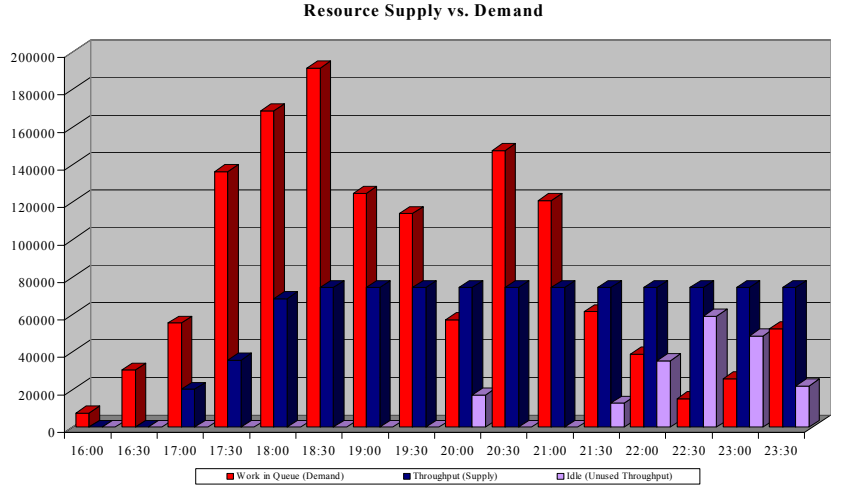

Figure 2: Graphical Representation of Simulation Results

The framework is designed not only for planning, but also for supporting tactical decision making and monitoring of actual environmental changes and events. The manager enters actual volumes, arrival profiles and attendance data into Excel spreadsheet and then runs the simulation model to assess the impacts and take necessary action. The planned and actual volumes and schedules are saved to the database and are used in the for forecasting module. The time to run the model is negligible.

\section{SIMULATION BASED SCHEDULE OPTIMIZATION}

As discussed above, the major challenge inherent to the proof shop environment is variability. Figure 3 illustrates variability in daily volumes, and the deviation between actual and planned volume on a given day. Analysis of historical data showed that this deviation could be as high as $15 \%$.

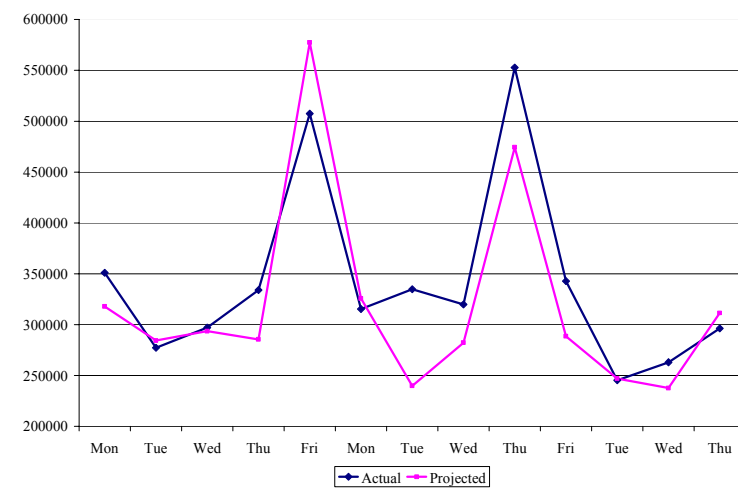

Figure 3: Variation in Total Volume for Consecutive Week Days at One Site. Y-axis - Number of Fields; $\mathrm{x}$-axis - Week Days

Figure 4 demonstrates variability in work arrival patterns and shows the percent of total volume arriving during a half hour time period during the day. While total volume has a statistically significant dependency on a number of factors - day of the week, month, holiday, etc. - the work arrival pattern follows the same basic shape: a peak between 3 and 4 PM, then a reduction between 4 PM and 6 PM that we are calling a valley and then a second, much higher peak that starts at $6 \mathrm{PM}$ and gradually reduces by $9 \mathrm{PM}$. This shape is dictated by transportation schedules and does not change drastically over time.

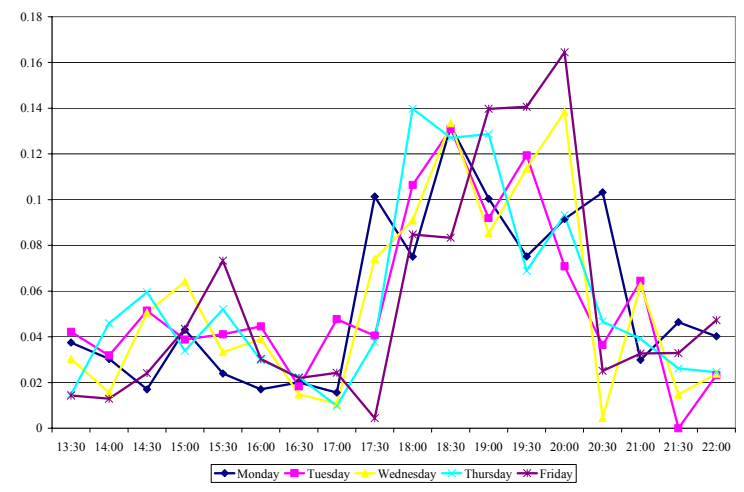

Figure 4: Work Arrival Patterns Monday through Friday. Y-axis - Percent of Total Volume; $x$-axis - Time of the Day

From the point of view of scheduling this drop in volume in the middle of the shift presents a major problem because over-staffing in the beginning of the shift can lead to excess capacity around 5 PM and under-staffing can result in missed deadlines. In Mabert (1979) the scheduling problem is formulated as constrained stochastic optimization, where the variables are shifts assigned to operators and the objective function is the probability of not having enough capacity to process expected work loads.

The approach proposed in this paper takes advantage of the following facts:

1. Operators are part time or hourly employees with a flexible shift pattern; the proposed solution assigns operators a start time for each business day at 15 minutes increments.

2. Use of simulation to estimate intermediate solutions allows one to incorporate in the model more details than a traditional mathematical model. In this case the simulation model contains rules defining individual lunch and ergonomic breaks, individual productivities, preferences and so forth.

3. The scheduled end of work is individual for operators and flexible; this fact allows creating a downward slope in total shop floor throughput at the end of the day that follows decreasing volumes (see Figure 4).

Given the limitations of accuracy in volume forecast the solution must not only be "optimal" for a given set of values defining an environment, but also robust and con- 
tain safeguards against unexpectedly high or low volumes and/or unfavorable work arrival patterns.

The proposed integrated simulation and heuristic approach to building a robust schedule is presented in Figure 5 . Based on the shape of the work arrival graph we introduce the notion of an unfavorable distribution. The most unfavorable scenario is one where less than expected work arrives in the first part of the shift and higher than expected volume is delivered in the second half of the shift exacerbating the "valleys" and the "peaks". Based on historical data we generate this "unfavorable scenario" by varying the forecast by $\pm 15 \%$.

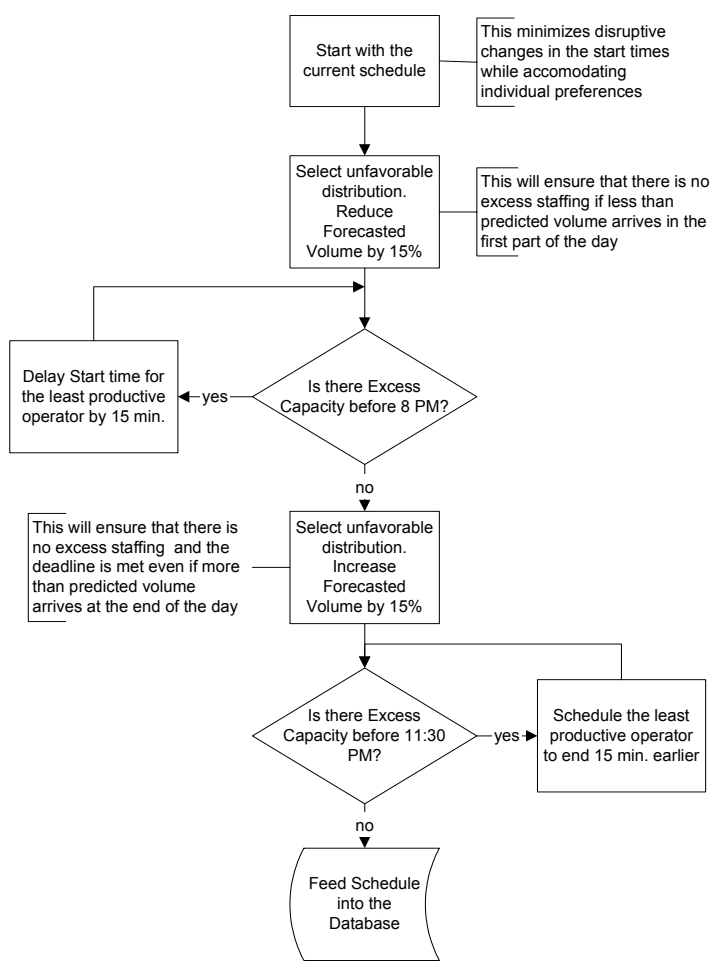

Figure 5: Heuristic for Building Optimized Robust Schedule

In the resulting schedule the starting times for the operators are selected in such a way that there is no excess capacity in the middle of the day even if less than predicted work is delivered later than usual. The end times are configured so that higher than predicted volumes could be processed before the deadline. These end times can later be adjusted according to the actual volumes and arrival pattern - the built-in simulator supports this functionality.

The current schedule serves as the initial solution that is improved in consequent iterations. This ensures that the operators' preferences concerning work days and start times are incorporated in the final solution.

Based on a retrospective analysis, productivity gains in excess of $30 \%$ could be realized using this approach. Gains vary by day of the week: maximum improvement was achieved on the days with light volumes.

\section{CONCLUSIONS}

Scheduling under uncertainty is a challenging task in which the decision maker is presented with a trade-off between over-staffing (excess capacity) and under-staffing with concomitant service and cost ramifications (missed deadlines and business opportunities). The proposed approach couples heuristic and simulation for building a robust optimized schedule that is robust even if the most unfavorable conditions come into play. If the situation is actually more favorable the user can quickly recalculate the estimates of overall productivity and completion time and adjust the schedule.

Using simulation to build intermediate solutions presented an opportunity to take a detailed view at the problem. In the described dynamic and highly variable environment even minor details, such as the timing of short ergonomic breaks, showed to influence the overall completion time.

Shop floor productivity and service levels as measured by completion time is estimated to increase on average by double digits due to the introduction of this planning and scheduling tool.

\section{REFERENCES}

Boyd, K. and V.A. Mabert. 1977. A Two Stage Forecasting Approach at Chemical Bank of New York for Check Processing. Journal of Banking Research (Summer): 101-107.

Mabert V.A. 1979. A case study of encoder shift scheduling under uncertainty. Management Science 25(7): 623-631.

Mallya, S.K. and A. Modi 2003. Operational Planning For Check Processing In Retail Banking Using Discrete Event Simulation. Presented at INFORMS Annual Meeting, Atlanta.

\section{AUTHOR BIOGRAPHIES}

Olga BAgATOUROVA, Ph. D. is a Process Design Engineer at the Bank of America, NA. She received her Ph.D. from the Institute of Management Sciences (Moscow) in 1980 and has since worked both in the Industry and Academia. Her research interests include mathematical and soft programming methods, artificial intelligence and data mining. She has extensive experience in development and implementation of scheduling methods and tools in manufacturing and quasi-manufacturing environments. Her e-mail address is <Olga.Bagatourova@bankofamerica. com>.

SUDHAKAR K. MALLYA is Senior Vice-President in Transaction Services at bank of America. He holds a Masters Degree in Industrial Engineering \& Operations Research from the University of Oklahoma in Norman and an MBA 
in Finance and Strategic Management from the University of Chicago. His interests include business strategy, forecasting, labor scheduling and the design, analysis and optimization of real world systems using stochastic modeling, computer simulation and related techniques. His e-mail address is <Sudhakar.K.Mallya@bankofamerica.com>. 